\section{Faltas ao trabalho por problemas de saúde na indústria}

\author{
Work days lost due to health problems \\ in industry
}

\author{
1 Serviço Social da Indústria, \\ Departamento Nacional, \\ Brasília, Brasil. \\ 2 Instituto de Saúde Coletiva \\ Universidade Federal da \\ Bahia, Brasília, Brasil. \\ Correspondência \\ S. R. T. Yano \\ Serviço Social da Indústria, \\ Departamento Nacional. \\ SBN Bl. C Q. 01, Ed. Roberto \\ Simonsen, $10 \underline{a}$ andar \\ UNISAUDE, Brasília, DF \\ 70040-903, Brasil. \\ sylvia.yano@gmail.com
}

\section{Abstract}

This cross-sectional study estimated the prevalence of work days lost due to health problems and associated factors among industrial workers. The study population was a simple random cluster sample of 3,403 workers from 16 to 65 years of age in the city of Salvador, Bahia State, Brazil. Data were collected with individual home interviews. Among industrial workers, one-year prevalence of work days lost to health problems was $12.5 \%$, of which $5.5 \%$ were directly work-related and $4.1 \%$ aggravated by work. There were no statistically significant differences when compared to other worker categories. Self-perceived workplace hazards, history of work-related injury, and poor self-rated health were associated with work days lost due to work-related injuries/diseases. The findings showed that work days lost are common among both industrial and non-industrial workers, thereby affecting productivity and requiring prevention programs.

Absenteeism; Working Environment; Occupational Diseases; Sick Leave; Industry
Sylvia Regina Trindade Yano ${ }^{1}$ Vilma Sousa Santana 2

\section{Introdução}

Faltas ao trabalho decorrentes de doenças e agravos à saúde (FTS) refletem incapacidades temporárias para o desempenho das atividades laborais. Demonstram não apenas a existência de problemas de saúde, mas também a sua gravidade, exceto quando resultam de gravidez, parto, ou quando compõem medidas para evitar a propagação de doenças transmissíveis. A incapacidade para o trabalho causada por problemas de saúde é de interesse não somente do trabalhador e dos profissionais de saúde, mas também das instituições responsáveis pelos benefícios de compensação salarial durante o afastamento, $\mathrm{e}$ das próprias empresas empregadoras.

Embora FTS sejam consideradas importante dimensão do estado de saúde de trabalhadores, estimativas de prevalência ou incidência de episódios ainda são raras. Estimativas de prevalência de FTS variam de 3,2\% nos Estados Unidos 1 a 7,6\% na Dinamarca 2, e 1,78\% entre jovens residentes no Canadá 2 . Observou-se também que pessoas com maior idade $3,4,5$, do sexo feminino 1,4,6,7, com menor número de anos de escolaridade 1,5,6,8, renda 4 ou nível socioeconômico 9 , tinham maior proporção de FTS em comparação com os referentes. Alguns fatores de risco para doenças crônicas como o hábito de fumar 6,10,11, consumo de bebidas alcoólicas 10, sobrepeso 4,10 , obesidade 5,10,11, saúde autopercebida ruim 4,11 e 
estresse ${ }^{4}$, também se associavam positivamente a FTS. Fatores ocupacionais relacionados ao aumento relativo da prevalência de FTS foram: ter ocupações menos qualificadas $1,4,8$, ou trabalho temporário ${ }^{8}$.

No Brasil, estudos epidemiológicos sobre FTS têm se concentrado em trabalhadores de saúde, especialmente de enfermagem, e funcionários públicos ou em empresas ou doenças específicas. Em um banco estatal, a prevalência de FTS foi estimada em $3,93 \%$ em 1998 , e $0,93 \%$ no ano de 2003, maior no sexo feminino, com estado civil separado, nos que tinham baixo grau de instrução e ocupações menos qualificadas. Idade e tempo de trabalho na empresa se associaram positivamente com afastamentos 12 . Com benefícios da Previdência Social, a prevalência de doenças respiratórias incapacitantes foi estimada em 9,9/10.000 trabalhadores segurados 13 .

$\mathrm{Na}$ indústria, o primeiro estudo sobre esta temática foi conduzido em uma empresa têxtil do Estado de São Paulo, entre 1970 e 1972. A prevalência de FTS para qualquer duração foi $40,8 \%$ entre homens, e 57,5 entre as mulheres 14. Especificamente com trabalhadores de indústrias metal-mecânicas, a prevalência de FTS por dor orofacial em um período de um ano foi 9,3\% 15 . Esses dados não permitem traçar a situação nacional relativa à FTS, e muito menos dos industriários, limitando-se aos empregados formais. Atividades industriais também compõem o mercado informal com um expressivo número de trabalhadores informais. Estudos de base populacional realizados com entrevistas domiciliares podem delinear um diferente perfil de afastamentos do trabalho que capturam o total de afastamentos, registrados ou não, em trabalhadores formais e informais. Neste estudo, com dados de um inquérito populacional, estima-se a prevalência de episódios de afastamentos para o trabalho, focalizando-se o trabalho na indústria. Identificam-se fatores associados de natureza sociodemográfica, ocupacional, fatores de risco para doenças crônicas e morbidade referida.

\section{Métodos}

Este estudo de desenho transversal foi conduzido com dados da etapa basal (Fase 1) de uma coorte iniciada em 2000 que focalizava acidentes de trabalho não fatais e condições de emprego. A população do estudo compreende residentes da cidade de Salvador, capital da Bahia, selecionados por amostragem aleatória, com um único estágio, de conglomerados definidos por superfície geográfica. Os conglomerados foram subáreas sorteadas com a ajuda de mapas de toda a área urbana. Em cada subárea, os limites geográficos foram checados e identificados pessoalmente por pesquisadores de campo, e listados todos os domicílios. Cada família foi registrada e uma pessoa chave de cada residência foi contatada para apresentação da investigação. Em seguida, agendavam-se entrevistas individuais, solicitando-se consentimento para participação.

Utilizaram-se questionários desenvolvidos especificamente para o estudo, definindo-se o conteúdo com base num mapa conceitual desenvolvido pela equipe, com base na literatura existente, em modelos teóricos, e nas próprias experiências pessoais das equipes de epidemiologia e etnografia envolvidas no projeto. A linguagem e viabilidade operacional dos instrumentos foram testadas em um estudo piloto. Os entrevistadores foram treinados e o seu trabalho supervisionado, por meio de reentrevistas pessoais ou telefônicas. Detalhes da metodologia empregada se encontram em outras publicações 16,17.

As variáveis de desfecho foram quatro, todas para o período compreendido pelos últimos 12 meses: (1) episódios de faltas ao trabalho por motivo de doença (FT-DG); (2) por doença causada pelo trabalho (FT-DT); (3) por doença agravada pelo trabalho (FT-DAT); e (4) por doença não relacionada ao trabalho (FT-DNRT). Tais variáveis foram construídas com perguntas que tinham respostas do tipo sim/não. As variáveis descritoras compreendem os aspectos sociodemográficos e ocupacionais relacionados à saúde e estilo de vida. As sociodemográficas são: sexo, idade; cor da pele definida pelo entrevistador, e categorizada em negros e não negros; nível socioeconômico foi definido com o número de bens da família ou equipamentos disponíveis no domicílio, especificamente: automóvel, computador, máquina de lavar, videocassete, toca-discos a laser, micro-ondas, máquina de lavar louça, telefone e casa de praia. $\mathrm{O}$ número total de itens foi categorizado em: baixo (menos de 3 itens); médio (3 a 5 itens) e alto (acima de 5 itens); nível de escolaridade categorizado em: alto (nível médio e superior, completo ou incompleto) e baixo (fundamental completo e incompleto); estado civil (casado/consensual, solteiro/outros); e, se tinha filhos ou não. Quanto às variáveis ocupacionais, levaram-se em conta: o ramo de atividade (indústria/outros ramos); horas de trabalho/dia (igual a ou menor que 8 horas, maior que 8 horas); rotatividade (mais de um emprego no último ano); formalidade do vínculo (registro em carteira de trabalho); percepção da ocupação como perigosa; e, treinamento ocupacional. Em relação às variáveis relacionadas à saúde, consideraram-se: o nível de saúde autopercebida, avaliando a percepção do estado de saúde como bom ou ruim; 
sentir-se feliz ou infeliz, sobrepeso definido pelo índice de massa corporal (IMC) acima de 24,9 a $29,9 \mathrm{~kg} / \mathrm{m}^{2}$; e, acidente de trabalho não fatal. As variáveis de estilo de vida foram: hábito de fumar e consumo abusivo de álcool, empregando-se critérios da DMS-IV, e as perguntas do Patient Health Questionnaire.

A prevalência foi a medida de morbidade, a razão de prevalência $(\mathrm{RP})$ foi a medida de associação. A estimativa estatística foi baseada no intervalo de $90 \%$ de confiança (IC90\%) pelo método de Mantel-Haenszel, adotando-se para a análise de dados o software estatístico SAS 9.2 (SAS Inst., Cary, Estados Unidos). Os resultados finais foram ajustados para o desenho da amostra, empregando-se rotinas desse software, surveyfreq e surveylogistic. O protocolo da pesquisa foi submetido e aprovado por uma Comissão de Ética em Pesquisa do Hospital Professor Edgard Santos da Universidade Federal da Bahia, atendendo a todas as recomendações legais. $\mathrm{O}$ anonimato e confidencialidade das informações foram mantidos nos registros dos dados e nas publicações. Toda a equipe da pesquisa recebeu no treinamento, instrução sobre os dispositivos e as responsabilidades éticas do estudo.

\section{Resultados}

A população deste estudo compreende trabalhadores remunerados, formais e informais, com idade entre 16 e 65 anos, totalizando 3.626 pessoas; $223(6,1 \%)$ recusaram participar do estudo, restando 3.403. Trabalhadores da indústria foram 512 (15,1\%). Os trabalhadores da indústria foram os que tinham trabalho em empresas manufatureiras, na construção civil, e em serviços públicos industriais.

A prevalência global de FT-DG foi de 13,5\%. Na Tabela 1, observa-se que entre os trabalhadores da indústria a prevalência de episódios de FT-DG foi de $12,5 \%, 5,5 \%$ para FT-DT, e $4,1 \%$ para as FT-DAT. A prevalência de FT-DNRT foi de $7 \%$ entre trabalhadores da indústria. Não houve diferenças entre as estimativas de prevalência desses desfechos, nos trabalhadores da indústria em comparação aos demais.

Os trabalhadores da indústria $(n=512)$ eram, na maioria, da construção civil $(52,1 \%)$, seguidos pela indústria manufatureira $(23,2 \%)$, comunicação (19,7\%), serviços elétricos (2,7\%), e outros $(2,1 \%)$. A maioria desses trabalhadores era do sexo masculino $(86,0 \%)$, tinha cor negra $(64,5 \%)$, era casada ou tinha relação consensual $(56,6)$, filhos $(68,8 \%)$, baixa escolaridade $(59,4 \%)$, e baixo nível socioeconômico $(58,1 \%)$. Eram também mais comuns os vínculos formais de trabalho (51,9\%), jornadas de trabalho com oito ou menos horas/dia $(64,5 \%)$, menor rotatividade $(90,6 \%)$, a percepção de que o trabalho era perigoso (59,5\%), e haviam recebido mais comumente treinamento para o trabalho $(55,2 \%)$ do que os demais trabalhadores. A prevalência de fumantes foi $19,5 \%$, maior que a do consumo abusivo de bebidas alcoólicas $(6,5 \%)$. O sobrepeso foi estimado em $33,4 \%$ da população de estudo. A saúde autopercebida como ruim foi referida por $8,2 \%$, e sentimentos de infelicidade por $7,6 \%$ dos participantes.

Trabalhadores da indústria tinham maior prevalência de FT-DG quando percebiam a atividade de trabalho como perigosa $(\mathrm{RP}=1,7$; IC90\%: 1,02-2,83), saúde autopercebida como ruim $(\mathrm{RP}=3,7$; IC90\%: 2,55-5,45) e haviam sofrido acidente de trabalho não fatal $(\mathrm{RP}=3,43$; IC90\%: $2,18-5,39)$ do que os grupos de comparação (Tabela 2). De modo semelhante, para a FT-DT, houve associação positiva entre percepção de atividade perigosa ( $\mathrm{RP}=2,50$; IC90\%: 0,92-6,78), de saúde autopercebida como ruim $(\mathrm{RP}=4,48$; IC90\%: 2,20-9,12) e de ter sofrido acidentes de trabalho não fatais (RP = 8,39; IC90\%: 5,01-14,06) do que os grupos referentes (Tabela 3). Na Tabela 4 , verifica-se que fatores associados à FT-DAT foram a saúde percebida como ruim, $(\mathrm{RP}=5,58$; IC90\%: 2,27-13,74) e história de acidentes de trabalho não fatais (RP = 5,58; IC90\%: 2,46-12,66). Para a FT-DNRT (Tabela 5), fatores associados foram o não treinamento para o trabalho ( $\mathrm{RP}=$ 2,43, IC90\%: 1,40-4,22), saúde percebida como ruim $(\mathrm{RP}=3,20$, IC90\%: 1,58-6,47), sentir-se infeliz ( $R P=2,42$, IC90\%: 1,0-5,89 e acidentes de trabalho não fatais (RP = 5,58, IC90\%: 2,46-12,66).

\section{Discussão}

Os resultados deste estudo revelam que na população total analisada, a prevalência de episódios de FTS em um ano foi maior para a FT-DG $(13,5 \%)$, reduzindo-se para $4,8 \%$ quando se tratava de FT-DT. A prevalência de FT-DAT é um pouco menor, de $3,8 \%$, enquanto para as doenças e agravos não relacionados ao trabalho, FT-DNRT foi $8,7 \%$. Encontrou-se também que não houve diferenças de prevalência de episódios de faltas ao trabalho, em todas as suas dimensões, entre trabalhadores da indústria e os demais ramos de atividade. Os fatores associados às faltas ao trabalho foram os mesmos para todos os desfechos, exceto para as doenças não relacionadas ao trabalho. Percepção de trabalho perigoso, ter sofrido acidente de trabalho não fatal e percepção da saúde ruim se associaram a faltas ao trabalho, tanto para problemas de saúde geral, como os 
Tabela 1

Prevalência das variáveis-desfecho, razões de prevalência (RP) e intervalos de $90 \%$ de confiança (IC90\%), brutas e ajustadas por sexo.

Salvador, Bahia, Brasil, 2000

\begin{tabular}{|c|c|c|c|c|c|c|c|c|}
\hline \multirow[t]{3}{*}{ Variáveis-desfecho } & \multicolumn{4}{|c|}{ Ramo de atividade } & \multirow{2}{*}{\multicolumn{2}{|c|}{$\begin{array}{l}\text { Associação bruta com } \\
\text { ramo de atividade }\end{array}$}} & \multirow{2}{*}{\multicolumn{2}{|c|}{$\begin{array}{c}\text { Associação com ramc } \\
\text { de atividade ajustada } \\
\text { por sexo }\end{array}$}} \\
\hline & \multicolumn{2}{|c|}{ Indústria manufatureira } & \multicolumn{2}{|c|}{ Outros } & & & & \\
\hline & $N=512$ & $\%$ & $N=2.891$ & $\%$ & RP & IC90\% & $\mathrm{RP}$ & IC90\% \\
\hline Faltou ao trabalho por doença & 64 & 12,5 & 395 & 13,7 & 1,00 & $095-1,02$ & 1,12 & $0,89-1,42$ \\
\hline $\begin{array}{l}\text { Faltou ao trabalho por doença causada } \\
\text { pelo trabalho }\end{array}$ & 28 & 5,5 & 135 & 4,7 & 1,01 & $0,98-1,04$ & 1,25 & $0,84-1,86$ \\
\hline $\begin{array}{l}\text { Faltou ao trabalho por doença agravada } \\
\text { pelo trabalho }\end{array}$ & 21 & 4,1 & 106 & 3,7 & 1,00 & $0,99-1,02$ & 1,28 & $0,88-1,86$ \\
\hline $\begin{array}{l}\text { Faltou ao trabalho por doença não relacionada } \\
\text { ao trabalho }\end{array}$ & 36 & 7,0 & 260 & 9,0 & 0,98 & $0,95-1,0$ & 1,03 & $0,74-1,43$ \\
\hline
\end{tabular}

relacionados ou agravados pelo trabalho. Distintamente, para as doenças não relacionadas ao trabalho, os fatores associados foram a falta de treinamento para o trabalho, o sentimento de infelicidade e também o sentir-se com saúde ruim. A morbidade autorreferida (sentir-se com saúde ruim e infeliz) se revelou o mais importante preditor potencial de episódios de falta ao trabalho, independentemente da natureza da relação entre o problema de saúde e o trabalho.

Os resultados deste estudo demonstram que as causas das faltas ao trabalho são mais frequentemente de natureza geral, não ocupacional, com prevalência três vezes maior do que as ausências motivadas por problemas de saúde resultantes da atividade de trabalho, na perspectiva da compreensão do trabalhador.

A população deste estudo abrange trabalhadores formais e informais, e para os últimos, não segurados, as faltas ao trabalho por motivo de doença cursam sem nenhum tipo de proteção social, e não são registradas. A identificação das faltas foi realizada apenas com as percepções dos sujeitos e os seus relatos de incapacidade para o trabalho, definida pela compreensão pessoal da natureza da relação com o trabalho 18 . É claro que a experiência do trabalhador informal em comparação com o formal, para a incapacidade para o trabalho é distinta, por suas presumíveis repercussões sociais, econômicas individuais e familiares. Os dados de morbidade geral e relacionada ao trabalho indicam a necessidade de que medidas de prevenção precisam se pautar pela promoção da saúde, focalizando problemas tanto ocupacionais como não ocupacionais e, assim, evitando futuras faltas ao trabalho.
Não foram encontradas diferenças entre as medidas de prevalência de qualquer um dos desfechos entre os trabalhadores da indústria em relação aos demais trabalhadores. Isso contrasta com dados de vários estudos 19,20,21 que mostram serem os trabalhadores da indústria mais comumente afetados por doenças e acidentes de trabalho. A não associação encontrada neste estudo pode ser resultado da singularidade dessa população, no que se refere à atividade industrial. Salvador é uma cidade cuja atividade econômica predominante é o turismo, o que resulta em grande dinamismo da sua atividade comercial e de serviços, e menos na indústria, atividade que se concentra em outros municípios que compõem sua região metropolitana. Os trabalhadores da indústria nessa população de estudo compõem-se, particularmente, de trabalhadores autônomos ou assalariados de pouca qualificação, especialmente da construção civil. Observações das atividades locais nas subáreas de estudo revelaram que além dessa, são comuns as microempresas cooperativadas ou informais, apoiadas por programas de fomento à microempreendedores, ou mesmo independentes. São comuns as indústrias de confecção de produtos alimentícios, bebidas, bolsas e calçados populares, frequentemente comercializados em suas próprias residências ou na vizinhança. Consequentemente, tal população não pode ser vista como representativa da média ou grande indústria, nas quais os fatores de risco para acidentes e doenças são comuns e, possivelmente, mais graves do que os existentes nas pequenas firmas informais. Estudos nacionais 17,22,23,24 vêm mostrando que a morbidade por acidentes de trabalho estimada em trabalhadores informais é 
Prevalência e razões de prevalência (RP) com intervalos de $90 \%$ de confiança (IC90\%) para associação entre variáveis sociodemográficas e episódios de falta ao trabalho por doença em geral (FT-DG) entre trabalhadores da indústria. Salvador, Bahia, Brasil, 2000.

\begin{tabular}{|c|c|c|c|c|}
\hline Variáveis & $N=512$ & FT-DG (\%) & RP & IC90\% \\
\hline \multicolumn{5}{|l|}{ Variáveis sociodemográficas } \\
\hline \multicolumn{5}{|l|}{ Sexo } \\
\hline Mulheres & 72 & 18,1 & 1,00 & - \\
\hline Homens & 440 & 11,6 & 0,64 & $0,40-1,04$ \\
\hline \multicolumn{5}{|l|}{ Idade (anos) } \\
\hline $16-21$ & 57 & 14,0 & 1,00 & - \\
\hline $22-40$ & 293 & 12,0 & 0,85 & $0,36-2,01$ \\
\hline $41-65$ & 162 & 13,0 & 0,92 & $0,44-1,93$ \\
\hline \multicolumn{5}{|l|}{ Cor da pele } \\
\hline Negro & 330 & 13,9 & 1,41 & $0,86-2,31$ \\
\hline Não negro & 182 & 9,9 & 1,00 & - \\
\hline \multicolumn{5}{|l|}{ Nível socioeconômico } \\
\hline Baixo & 298 & 15,1 & 1,16 & $0,58-2,30$ \\
\hline Médio & 153 & 7,2 & 0,55 & $0,23-1,31$ \\
\hline Alto & 61 & 13,1 & 1,00 & - \\
\hline \multicolumn{5}{|l|}{ Escolaridade } \\
\hline Alta & 208 & 11,1 & 1,00 & - \\
\hline Baixa & 304 & 13,5 & 1,20 & $0,78-1,90$ \\
\hline \multicolumn{5}{|l|}{ Estado civil } \\
\hline Casado/Consensual & 290 & 12,1 & 1,00 & - \\
\hline Solteiro/Outros & 222 & 13,1 & 1,08 & $0,69-1,69$ \\
\hline Tem filhos & 350 & 11,7 & 0,81 & $0,51-1,29$ \\
\hline \multicolumn{5}{|l|}{ Variáveis ocupacionais } \\
\hline \multicolumn{5}{|l|}{ Horas de trabalho/dia } \\
\hline$\leq 8$ & 330 & 12,4 & 1,00 & - \\
\hline$>8$ & 182 & 12,6 & 1,02 & $0,63-1,40$ \\
\hline Alta rotatividade (> 1 emprego/ano) & 48 & 6,3 & 0,47 & $0,17-1,29$ \\
\hline O vínculo de trabalho é informal & 240 & 10,4 & 0,73 & $0,51-1,03$ \\
\hline Percebe a atividade como perigosa & 301 & 15,0 & 1,70 & $1,02-2,83$ \\
\hline Recebeu treinamento ocupacional & 281 & 14,6 & 1,45 & $0,98-2,13$ \\
\hline \multicolumn{5}{|l|}{ Variáveis do estilo de vida } \\
\hline Fuma & 100 & 9,0 & 0,67 & $0,39-1,17$ \\
\hline Consome abusivamente álcool & 33 & 9,1 & 0,71 & $0,29-1,77$ \\
\hline \multicolumn{5}{|l|}{ Variáveis relacionadas à saúde } \\
\hline Sente-se com saúde ruim & 42 & 38,1 & 3,73 & $2,55-5,45$ \\
\hline Sente-se infeliz & 39 & 20,5 & 1,73 & $0,92-3,27$ \\
\hline Sobrepeso & 166 & 12,7 & 0,97 & $0,61-1,57$ \\
\hline Acidentes de trabalho não fatais & 42 & 35,7 & 3,43 & $2,18-5,39$ \\
\hline
\end{tabular}

menor do que entre os formais, ao contrário do que têm revelado pesquisas desenvolvidas em países industrializados 25.

É difícil a comparação com episódios de faltas ao trabalho de base populacional, pois a maioria dos estudos foi conduzida com dados de sistemas de seguridade social, e mesmo entre eles, os critérios de elegibilidade variam, a exemplo do número de dias requeridos para o registro, e o recebimento de benefícios de compensação. Achados de um estudo transversal realizado nos Estados Unidos, com dados coletados em entrevistas, mostraram que a prevalência de episódios de faltas ao trabalho por doenças e acidentes foi 
Prevalência e razões de prevalência (RP) com intervalos de $90 \%$ de confiança (IC90\%) para associação entre variáveis sociodemográficas e episódios de falta ao trabalho por doença relacionada ao trabalho (FT-DT) entre trabalhadores da indústria. Salvador, Bahia, Brasil, 2000.

\begin{tabular}{|c|c|c|c|c|}
\hline Variáveis & $N=512$ & FT-DT (\%) & $\mathrm{RP}$ & IC90\% \\
\hline \multicolumn{5}{|l|}{ Variáveis sociodemográficas } \\
\hline \multicolumn{5}{|l|}{ Sexo } \\
\hline Mulheres & 72 & 5,6 & 1,00 & - \\
\hline Homens & 440 & 5,5 & 0,98 & $0,41-2,33$ \\
\hline \multicolumn{5}{|l|}{ Idade (anos) } \\
\hline $16-21$ & 57 & 8,8 & 1,00 & - \\
\hline $22-40$ & 293 & 3,8 & 0,43 & $0,13-1,40$ \\
\hline $41-65$ & 162 & 7,4 & 0,84 & $0,29-2,46$ \\
\hline \multicolumn{5}{|l|}{ Cor da pele } \\
\hline Negro & 330 & 5,8 & 1,16 & $0,73-1,86$ \\
\hline Não negro & 182 & 5,0 & 1,00 & - \\
\hline \multicolumn{5}{|l|}{ Nível socioeconômico } \\
\hline Baixo & 298 & 6,4 & 1,95 & $0,66-5,79$ \\
\hline Médio & 153 & 4,6 & 1,40 & $0,42-4,63$ \\
\hline Alto & 61 & 3,3 & 1,00 & - \\
\hline \multicolumn{5}{|l|}{ Escolaridade } \\
\hline Alta & 208 & 3,9 & 1,00 & - \\
\hline Baixa & 304 & 6,6 & 1,71 & $0,94-3,10$ \\
\hline \multicolumn{5}{|l|}{ Estado civil } \\
\hline Casado/Consensual & 290 & 5,5 & 1,00 & - \\
\hline Solteiro/Outros & 222 & 5,4 & 0,97 & $0,49-1,97$ \\
\hline Tem filhos & 350 & 6,0 & 1,36 & $0,54-3,46$ \\
\hline \multicolumn{5}{|l|}{ Variáveis ocupacionais } \\
\hline \multicolumn{5}{|l|}{ Horas de trabalho/dia } \\
\hline$\leq 8$ & 330 & 5,5 & 1,00 & - \\
\hline$>8$ & 182 & 5,5 & 1,01 & $0,51-1,98$ \\
\hline Alta rotatividade (> 1 emprego/ano) & 48 & 2,1 & 0,36 & $0,06-2,09$ \\
\hline O vínculo de trabalho é informal & 240 & 4,2 & 0,63 & $0,32-1,27$ \\
\hline Percebe a atividade como perigosa & 301 & 7,3 & 2,50 & $0,92-6,78$ \\
\hline Recebeu treinamento ocupacional & 281 & 5,0 & 0,81 & $0,48-1,37$ \\
\hline \multicolumn{5}{|l|}{ Variáveis do estilo de vida } \\
\hline Fuma & 100 & 3,0 & 0,49 & $0,19-1,18$ \\
\hline Consome abusivamente álcool & 123 & 4,1 & 0,54 & $0,10-3,01$ \\
\hline \multicolumn{5}{|l|}{ Variáveis relacionadas à saúde } \\
\hline Sente-se com saúde ruim & 42 & 19,1 & 4,48 & $2,20-9,12$ \\
\hline Sente-se infeliz & 99 & 5,13 & 0,93 & $0,27-3,24$ \\
\hline Sobrepeso & 166 & 7,2 & 1,50 & $0,67-3,33$ \\
\hline Acidentes de trabalho não fatais & 42 & 28,6 & 8,39 & $5,01-14,60$ \\
\hline
\end{tabular}

menor do que a estimada neste estudo, variando de $4,7 \%$ entre negros e homens hispânicos brancos a 3,2\% entre brancos não hispânicos, e variou de $3 \%$ entre as mulheres negras para aproximadamente $2,2 \%$ entre as brancas não hispânicas 1. Embora Smeby et al. 4 também tenham utilizado entrevistas telefônicas com uma amostra da população geral da Noruega, a sua população de estudo tinha mais idade, com média acima de 40 anos, e a prevalência foi de 12,5\% entre as mulheres e 8,7\% entre os homens, para o período de um ano. Esses dados são próximos às estimativas deste estudo, para a população de ambos os sexos. 
Prevalência e razões de prevalência (RP) com intervalos de 90\% de confiança (IC90\%) para associação entre variáveis sociodemográficas e episódios de falta ao trabalho por doença agravada pelo trabalho (FT-DAT) entre trabalhadores da indústria. Salvador, Bahia, Brasil, 2000.

\begin{tabular}{|c|c|c|c|c|}
\hline Variáveis & $N=512$ & FT-DAT (\%) & RP & IC90\% \\
\hline \multicolumn{5}{|l|}{ Variáveis sociodemográficas } \\
\hline \multicolumn{5}{|l|}{ Sexo } \\
\hline Mulheres & 72 & 6,9 & 1,00 & - \\
\hline Homens & 439 & 3,6 & 0,52 & $0,21-1,31$ \\
\hline \multicolumn{5}{|l|}{ Idade (anos) } \\
\hline $16-21$ & 57 & 8,8 & 1,00 & - \\
\hline $22-40$ & 293 & 2,7 & 0,31 & $0,09-1,07$ \\
\hline $41-65$ & 161 & 5,0 & 0,57 & $0,19-1,73$ \\
\hline \multicolumn{5}{|l|}{ Cor da pele } \\
\hline Negro & 330 & 4,5 & 1,37 & $0,63-3,00$ \\
\hline Não negro & 181 & 3,3 & 1,00 & - \\
\hline \multicolumn{5}{|l|}{ Nível socioeconômico } \\
\hline Baixo & 298 & 4,7 & 0,72 & $0,30-1,74$ \\
\hline Médio & 152 & 2,0 & 0,30 & $0,09-1,03$ \\
\hline Alto & 61 & 6,6 & 1,00 & - \\
\hline \multicolumn{5}{|l|}{ Escolaridade } \\
\hline Alta & 208 & 2,9 & 1,00 & - \\
\hline Baixa & 303 & 5,0 & 1,72 & $0,83-3,54$ \\
\hline \multicolumn{5}{|l|}{ Estado civil } \\
\hline Casado/Consensual & 289 & 4,5 & 1,00 & - \\
\hline Solteiro/Outros & 222 & 3,6 & 0,80 & $0,33-1,92$ \\
\hline Tem filhos & 349 & 4,3 & 1,37 & $0,62-3,01$ \\
\hline \multicolumn{5}{|l|}{ Variáveis ocupacionais } \\
\hline \multicolumn{5}{|l|}{ Horas de trabalho/dia } \\
\hline$\leq 8$ & 329 & 4,0 & 1,00 & - \\
\hline$>8$ & 182 & 4,0 & 1,11 & $0,46-2,71$ \\
\hline Alta rotatividade (> 1 emprego/ano) & 48 & - & 1,05 & - \\
\hline O vínculo de trabalho é informal & 240 & 3,3 & 0,72 & $0,40-1,30$ \\
\hline Percebe a atividade como perigosa & 301 & 5,3 & 2,18 & $0,85-5,62$ \\
\hline Recebeu treinamento ocupacional & 281 & 4,3 & 1,08 & $0,53-2,18$ \\
\hline \multicolumn{5}{|l|}{ Variáveis do estilo de vida } \\
\hline Fuma & 100 & 2,0 & 0,43 & $0,14-1,34$ \\
\hline Consome abusivamente álcool & 123 & 0,8 & 0,72 & $0,14-3,72$ \\
\hline \multicolumn{5}{|l|}{ Variáveis relacionadas à saúde } \\
\hline Sente-se com saúde ruim & 42 & 16,7 & 5,58 & $2,27-13,74$ \\
\hline Sente-se infeliz & 39 & 7,7 & 2,01 & $0,73-5,53$ \\
\hline Sobrepeso & 166 & 4,22 & 1,00 & $0,43-2,32$ \\
\hline Acidentes de trabalho não fatais & 42 & 16,7 & 5,58 & $2,46-12,66$ \\
\hline
\end{tabular}

Fatores associados às faltas ao trabalho na indústria manufatureira encontrados neste estudo demonstram a importância dos fatores de risco conhecidos pelos trabalhadores, que os levaram a considerar sua atividade como perigosa. Consistentemente, os acidentes de trabalho que contribuem com a maior parcela dos benefícios por motivo de doenças na Previdência, também apareceram como fator associado. A saúde percebida como ruim também se revelou como indicador importante de faltas ao trabalho, corroborando o achado de Smeby et al. 4 .

Um aspecto importante não abordado neste estudo é o impacto das faltas ao trabalho na 
Prevalência e razões de prevalência (RP) com intervalos de 90\% de confiança (IC90\%) para associação entre variáveis sociodemográficas e episódios de falta ao trabalho por doença não relacionada ao trabalho (FT-DNRT) entre trabalhadores da indústria. Salvador, Bahia, Brasil, 2000.

\begin{tabular}{|c|c|c|c|c|}
\hline Variáveis & $N=512$ & FT-DNRT (\%) & RP & IC90\% \\
\hline \multicolumn{5}{|l|}{ Variáveis sociodemográficas } \\
\hline \multicolumn{5}{|l|}{ Sexo } \\
\hline Mulheres & 72 & 12,5 & 1,00 & - \\
\hline Homens & 440 & 6,1 & 0,49 & $0,28-0,86$ \\
\hline \multicolumn{5}{|l|}{ Idade (anos) } \\
\hline $16-21$ & 57 & 5,3 & 1,00 & - \\
\hline $22-40$ & 293 & 8,2 & 1,56 & $0,45-5,37$ \\
\hline $41-65$ & 162 & 5,6 & 1,06 & $0,31-3,56$ \\
\hline \multicolumn{5}{|l|}{ Cor da pele } \\
\hline Negro & 330 & 8,2 & 1,65 & $0,81-3,39$ \\
\hline Não negro & 182 & 5,0 & 1,00 & - \\
\hline \multicolumn{5}{|l|}{ Nível socioeconômico } \\
\hline Baixo & 298 & 8,7 & 0,89 & $0,36-2,19$ \\
\hline Médio & 153 & 2,6 & 0,27 & $0,08-0,84$ \\
\hline Alto & 61 & 9,8 & 1,00 & - \\
\hline \multicolumn{5}{|l|}{ Escolaridade } \\
\hline Alta & 208 & 7,2 & 1,00 & - \\
\hline Baixa & 304 & 6,9 & 0,96 & $0,57-1,60$ \\
\hline \multicolumn{5}{|l|}{ Estado civil } \\
\hline Casado/Consensual & 290 & 6,6 & 1,00 & - \\
\hline Solteiro/Outros & 222 & 7,7 & 1,17 & $0,73-1,88$ \\
\hline Tem filhos & 350 & 5,7 & 0,57 & $0,33-0,99$ \\
\hline \multicolumn{5}{|l|}{ Variáveis ocupacionais } \\
\hline \multicolumn{5}{|l|}{ Horas de trabalho/dia } \\
\hline$\leq 8$ & 330 & 7,0 & 1,00 & - \\
\hline$>8$ & 182 & 7,1 & 1,02 & $0,54-1,95$ \\
\hline Alta rotatividade (> 1 emprego/ano) & 48 & 4,2 & 0,57 & $0,17-1,85$ \\
\hline O vínculo de trabalho é informal & 240 & 6,3 & 0,81 & $0,50-1,30$ \\
\hline Percebe a atividade como perigosa & 301 & 7,6 & 1,31 & $0,82-2,07$ \\
\hline Recebeu treinamento ocupacional & 281 & 9,6 & 2,43 & $1,40-4,22$ \\
\hline \multicolumn{5}{|l|}{ Variáveis do estilo de vida } \\
\hline Fuma & 100 & 6,0 & 0,82 & $0,36-1,88$ \\
\hline Consome abusivamente álcool & 123 & 1,6 & 0,85 & $0,27-2,65$ \\
\hline \multicolumn{5}{|l|}{ Variáveis relacionadas à saúde } \\
\hline Sente-se com saúde ruim & 42 & 19,1 & 3,20 & $1,58-6,40$ \\
\hline Sente-se infeliz & 39 & 15,4 & 2,42 & $1,00-5,88$ \\
\hline Sobrepeso & 166 & 5,4 & 0,66 & $0,37-1,19$ \\
\hline Acidentes de trabalho não fatais & 42 & 16,7 & 1,02 & $0,39-2,67$ \\
\hline
\end{tabular}

produtividade e as consequências econômicas, que podem se constituir em argumentos significativos no apoio a medidas de prevenção de riscos e na promoção da saúde. Mesmo quando trabalhadores são substituídos, existem os custos com contratação de substitutos, treinamento, redução do ritmo da produção, dentre outros 26 .
Os achados deste estudo devem ser considerados valendo-se de suas limitações. O desenho é transversal e não é possível a garantia de antecedência temporal dos fatores em relação aos desfechos. A análise foi exploratória, voltada para a identificação de hipóteses, a serem analisadas posteriormente. Um aspecto importante 
a ser focalizado é o presenteísmo, ou especificamente para os trabalhadores informais, o tipo de rearranjos familiares e o papel do apoio social para garantia da sobrevivência em caso de incapacidade para o trabalho. Além disso, os dados são autorreferidos pelos trabalhadores e se sustentam em suas percepções e julgamentos subjetivos, o que pode estar sujeito a vieses vários, apesar de evidências em contrário 27.
As perdas representaram apenas $6,1 \%$ dos elegíveis e não há indicação de que possam ter afetado as conclusões do estudo.

Com essas informações, a equipe de saúde, segurança e recursos humanos pode prevenir, diminuir ou acabar com os casos de ausências no trabalho causadas por doenças relacionadas com o trabalho.

\section{Resumo}

Neste estudo transversal, estima-se a prevalência de episódios de faltas ao trabalho por motivo de saúde (FTS), e fatores associados, focalizando o trabalho na indústria. A população do estudo foi uma amostra aleatória por conglomerado de 3.403 trabalhadores de 16 a 65 anos residentes em Salvador, Bahia, Brasil. Dados foram obtidos em entrevistas domiciliares. Entre os trabalhadores da indústria, a prevalência em um ano de FTS em geral, foi 12,5\%, 5,5\% para FTS relacionados ao trabalho, e 4,1\% para FTS agravados pelo trabalho. Não houve diferenças estatisticamente significantes em relação aos demais trabalhadores. Fatores associados à FTS foram percepção de perigo no trabalho, história de acidente de trabalho, e saúde autopercebida como ruim. Os resultados indicam que a ocorrência de faltas ao trabalho é comum, tanto na indústria como nos demais ramos de atividade, podem afetar a produtividade, o que requer programas de prevenção de agentes de risco em locais de trabalho.

Absenteísmo; Ambiente de Trabalho; Doenças do Trabalho; Licença Médica; Indústria

\section{Colaboradores}

S. R. T. Yano e V. S. Santana contribuíram igualmente na elaboração do artigo. 


\section{Referências}

1. Strong LL, Zimmerman FJ. Occupational injury and absence from work among African American, Hispanic, and Non-Hispanic White workers in the National Longitudinal Survey of Youth. Am J Public Health 2005; 95:1226-32.

2. Mustard C, Cole D, Shannon H, Pole J, Sullivan T, Allingham R. Declining trends in work-related morbidity and disability, 1993-1998: a comparison of survey estimates and compensation insurance claims. Am J Public Health 2003; 93:1283-6.

3. Tsai SP, Gilstrap EL, Colangelo TA, Menard AK, Ross CE. Illness absence at an oil refinary and petrochemical plant. J Occup Environ Med 1997; 39:455-62.

4. Smeby L, Bruusgaard D, Claussen B. Sickness absence: could gender divide be explained by occupation, income, mental distress and health? Scand J Public Health 2009; 37:674-81.

5. Lidwall U, Bergendorff S, Voss M, Marklund S. Long-term sickness absence: changes in risk factors and the population at risk. Int J Occup Med Environ Health 2009; 22:157-68.

6. Lund T, Csonka A. Risk factors in health, work environment, smoking status, and organizational context for work disability. Am J Ind Med 2003; 44:492-501.

7. Rasmussen M. Work first: explaining the drop in disability claims in Denmark. In: Kemp PA, Sundén A, Tauritz BB, editors. Sick societies? Trends in disability benefits in post-industrial welfare states. Geneva: International Social Security Association; 2006. p. 51-77.

8. Lu X, Laaksonen M, Aittomäki A, Leino-Arjas P, Rahkonen O, Saatamoinen P, et al. Sickness absence, employment history, and high-risk employees: a 10-year longitudinal study. J Occup Environ Med 2010; 52:913-9.

9. Christensen KB, Labriola M, Lund T, Kivimäki M. Explaining the social gradient in long-term sickness absence: a prospective study of Danish employees. J Epidemiol Community Health 2008; 62:181-3.

10. Laaksonen LM, Piha K, Martikainen P, Rahkonen E. Health-related behaviours and sickness absence from work. Occup Environ Med 2009; 66:840-7.

11. Lund T, Christensen KB, Vaez M, Labriola M, Josephson M, Villadsen E, et al. Differences in sickness absence in Sweden and Denmark: the cross national HAKNAK study. Eur J Public Health 2008; 19:343-9.

12. Silva LS, Pinheiro TMM, Sakurai E. Perfil do absenteísmo em um banco estatal em Minas Gerais: análise no período de 1998 a 2003. Ciênc Saúde Coletiva 2008; 13:2049-58.

13. Ildefonso SAG, Barbosa-Branco A, AlbuquerqueOliveira PR. Prevalência de benefícios de seguridade social temporários devido à doença respiratória no Brasil. J Bras Pneumol 2009; 35:44-53.
14. Nogueira DP, Laurenti R. Absenteísmo por doença em mulheres. Rev Saúde Pública 1975; 9:393-9.

15. Lacerda JT, Traebert J, Zambenedetti ML. Dor orofacial e absenteísmo em trabalhadores da indústria metalúrgica e mecânica. Saúde Soc 2008; 17:182-91.

16. Santana VS, Maia AP, Carvalho C, Luz G. Acidentes de trabalho não fatais: diferenças de gênero e tipo de contrato. Cad Saúde Pública 2003; 19:481-93.

17. Santana VS, Loomis D. Informal jobs and nonfatal occupational injuries. Ann Occup Hyg 2004; 48:147-57.

18. Iriart JAB, Oliveira RP, Xavier SS, Costa AM, Araújo GR, Santana VS. Representações do trabalho informal e dos riscos à saúde entre trabalhadoras domésticas e trabalhadores da construção civil. Ciênc Saúde Coletiva 2008; 13:165-74.

19. Mital A, Pennathur A, Kansal A. Nonfatal occupational injuries in the United States. Part I - overall trends and data summaries. Int J Ind Ergon 1999; 25:109-29.

20. Stout NA, Linn HI. Occupational injury prevention research: progress and priorities. Inj Prev 2002; 8:9-14.

21. Santana VS, Nobre LC, Waldvogel M. Acidentes de trabalho no Brasil entre 1994 e 2004: uma revisão. Ciênc Saúde Coletiva 2005; 10:841-55.

22. Barata R, Ribeiro MC. Pesquisa condições de vida - 1994: componente acidentes de trabalho e doenças profissionais. São Paulo: Fundação Seade; 1998.

23. Cordeiro R, Prestes SCC, Clemente APG, Diniz CS, Sakate M, Donalisio MR. Incidência de acidentes do trabalho não fatais em localidade do Sudeste do Brasil. Cad Saúde Pública 2006; 22:387-93.

24. Corrêa-Filho H, Cugliari L, Gaspar AAC, Loureiro JF, Siqueira CE. Epidemiological surveillance of informal workers' health in two cities in Southeastern Brazil - the experience of the TRAPP-TRAPPURA Projects. Int J Occup Environ Health 2010; 16:36-46.

25. Commission on Social Determinants of Health, World Health Organization. Employment conditions and health inequities. Final report, 2007. http://www.who.org (acessado em 14/Out/2010).

26. Rikhardsson PM, Impgaard M. Corporate cost of occupational accidents: an activity-based analysis. Accid Anal Prev 2004; 36:173-82.

27. Mortelmans AK, Donceel P, Lahaye D, Bulterys S. Work-related sickness absences and mandatory occupational health surveillance. Occup Med 2008; 58:464-7.

Recebido em 17/Fev/2011

Versão final reapresentada em 21/Dez/2011

Aprovado em 31/Jan/2012 